\title{
Comportamiento de la lombriz roja (Eisenia spp.) en sistemas de vermicompostaje de residuos orgánicos
}

\section{Red worm behavior (Eisenia spp.) in vermicomposting systems of organic residues}

\author{
Mamani-Mamani Gladys, Mamani-Pati Francisco*, Sainz-Mendoza Humberto, Villca-Huanaco René
}

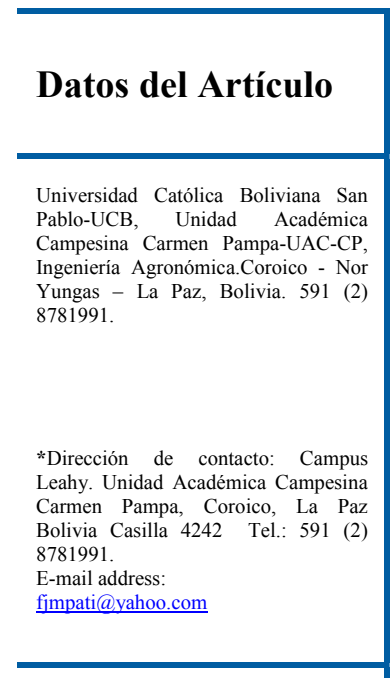

\section{Palabras clave:}

Vermicompost,

Eisenia, lombriz roja

estiércol, sustrato,

residuos sólidos,

basura, fitotoxicidad

\section{Resumen}

El presente trabajo evalúa el comportamiento de la lombriz roja (Eisenia spp.) en dos ambientes (Carpa Solar y Ambiente Natural) utilizando como sustrato dos tipos de residuos sólidos orgánicos (Estiércol Vacuno = EV y Restos de Cocina $=\mathrm{RC}$ ) en Predios de la Estación Experimental de la carrera de Ingeniería Agronómica de la Unidad Académica Campesina Carmen Pampa, Dependiente de la Universidad Católica Boliviana, en la Comunidad Carmen Pampa, Nor Yungas, Departamento de La Paz. Se adoptó un diseño bifactorial con tres repeticiones y los datos se sometieron al análisis de varianza (ANVA). El mayor número de cápsulas (cocones) se obtuvo en el ambiente Carpa Solar siendo para EV de 64 y RC de 41 cápsulas. En cuanto al porcentaje de eclosión, en el ambiente Carpa Solar se registró $100 \%$ de eclosión en EV y $96 \%$ en RC. Por consiguiente, el mayor número de lombrices nacidas ocurrió en el ambiente Carpa Solar, siendo 2 lombrices por cápsula para EV y 3.5 lombrices por cápsula para RC. La mayor tasa de crecimiento de lombrices inmaduras (no cliteladas) se presentó en el ambiente Carpa Solar resultando para EV de 123 individuos y RC de 16 lombrices. Por lo tanto, la menor tasa de mortalidad entre ambientes se produce en ambiente Carpa Solar con $3.90 \%$ de mortalidad para EV y RC con $88.64 \%$. Por otro lado, el mayor número de lombrices cliteladas se encuentra en Ambiente Natural siendo en EV de 15 individuos y RC con 21 lombrices cliteladas. En cuanto a la biomasa de lombrices no cliteladas, el mejor resultado se registró en ambiente Carpa Solar obteniéndose para EV $1.41 \mathrm{~g}$ y para RC $0.185 \mathrm{~g}$. Sin embargo, la mayor biomasa total de lombrices cliteladas se obtuvo en RC 7.98 g para el ambiente Carpa Solar frente a $6.93 \mathrm{~g}$ en el Ambiente Natural. El grado de fitotoxicidad del vermicompost obtenido de EV en los dos ambientes fue bajo, presentando $66.6 \%$ de germinación, ya el producto obtenido de RC presentó mayor fitotoxicidad en ambos ambientes. Con respecto al análisis de macronutrientes en los vermicompost obtenidos, se determinó un contenido de 2.45 y $2.31 \%$ de nitrógeno; 500 y $220 \mathrm{mg} \mathrm{kg}^{-1}$ de fósforo; 27.43 y $2.76 \mathrm{cmol}$ (+) $\mathrm{kg}^{-1}$ de sustrato seco en los sustratos $\mathrm{RC}$ y EV respectivamente.

(C) 2012. Journal of the Selva Andina Research Society. Bolivia. Todos los derechos reservados.

\section{Abstract}

This work evaluates the behavior of the vermicomposting red worm (Eisenia spp.) in two environments (greenhouse and unsheltered) and with two solid organic substrates (cow manure $=\mathrm{CM}$ and kitchen waste $=\mathrm{KW}$ ) in the zone of Carmen Pampa, Nor Yungas Province, Department of La Paz - Bolivia, using a factorial design with two factors and three repetitions (ANOVA analysis). The largest number of cocoons was found in the greenhouse treatment, with 64 in $\mathrm{CM}$ and 41 in $\mathrm{KW}$. Cocoon viability was also greatest in the greenhouse treatment, at $100 \%$ for $\mathrm{CM}$ and $96 \%$ for KW. Similarly, the greatest number of worms hatched from cocoons was in the greenhouse treatment, with 2
Recibido Abril, 2012 Devuelto Mayo 2012
Aceptado Julio, 2012 .

Disponible en línea, Agosto 2012. 


\begin{tabular}{|c|c|}
\hline $\begin{array}{l}\text { Vermicompost, } \\
\text { Eisenia, red worms, } \\
\text { manure, substrate, } \\
\text { solid residues, } \\
\text { solid waste. } \\
\text { phytotoxicity }\end{array}$ & $\begin{array}{l}\text { immature worms for } \mathrm{CM} \text { and } 3.5 \text { for } \mathrm{KW} \text {, and the greatest number of immature worms was registered in the } \\
\text { greenhouse treatment with } 123 \text { individuals in } \mathrm{CM} \text { and } 16 \mathrm{in} \mathrm{KW} \text {. The lowest mortality rate due to environment was in } \\
\text { the greenhouse treatment, with } 3.90 \% \text { mortality with } \mathrm{CM} \text { and } 88.64 \% \text { with } \mathrm{KW} \text {. The greatest number of mature } \\
\text { worms (with clitella) was in the unsheltered treatment, with } 15 \mathrm{in} \mathrm{CM} \text { and } 21 \text { in } \mathrm{KW} \text {. The greatest biomass of } \\
\text { immature worms was found in the greenhouse treatment, with } 1.41 \mathrm{~g} \text { of worms for } \mathrm{CM} \text { and } 0.185 \mathrm{~g} \text { for } \mathrm{KW} \text {; however, } \\
\text { the greatest biomass of mature worms was in the } \mathrm{KW} \text { treatment, with } 7.98 \mathrm{~g} \text { for the greenhouse treatment and } 6.93 \mathrm{~g}\end{array}$ \\
\hline $\begin{array}{l}\text { Editado por: } \\
\text { "Selva Andina } \\
\text { Research } \\
\text { Society" }\end{array}$ & $\begin{array}{l}\text { exhibiting a } 66.6 \% \text { rate of germination, and the opposite was true for } \mathrm{KW} \text {, which was the most toxic in both } \\
\text { environments. Macronutrient content in vermicompost obtained was: nitrogen at } 2.45 \% \text { and } 2.31 \% \text {, phosphorus at } 500 \\
\mathrm{mg} \mathrm{kg}^{-1} \text { and } 220 \mathrm{mg} \mathrm{kg}^{-1} \text { and potassium at } 27.43 \text { and } 2.76 \mathrm{cmol}_{(+)} \mathrm{kg}^{-1} \text { of dry substrate in } \mathrm{KW} \text { and } \mathrm{CM} \text { respectively. }\end{array}$ \\
\hline
\end{tabular}

\section{Introducción}

La generación de residuos sólidos o "basura" en las principales ciudades de Bolivia varía entre 0.3 a $0.7 \mathrm{~kg} /$ habitante/día de los cuales una gran proporción (de hasta el 70\%) corresponde a la fracción orgánica o fermentable cuyo destino final son botaderos o rellenos sanitarios (SNCSB 2000). No obstante, en el ámbito rural se enfrentan serias dificultades para resolver la problemática del manejo y disposición final, con el consiguiente riesgo de convertirlos en focos de contaminación ambiental y proliferación de patógenos y vectores que amenazan la salud pública.

La situación descrita obliga a buscar soluciones técnicas entre las cuales existen algunas de gran complejidad y elevado costo. Como alternativa, surgen los sistemas biológicos de tratamiento como el compostaje y vermicompostaje (compostaje con lombrices) los cuales promueven la reducción de los volúmenes, mitigación de la toxicidad y patogenicidad de los residuos sólidos generados. La tecnología del vermicompostaje, se basa en el hábito de alimentación detritívoro de algunas especies de lombrices (Annelida,
Lumbricidae) entre las que destaca el género Eisenia, organismos capaces de colonizar una gran variedad de sustratos orgánicos. Su acción sumada a la microbiana, promueve una efectiva degradación de la fracción orgánica contenida en los residuos sólidos domésticos o domiciliarios e inclusive agroindustriales (Sainz et al 2000, Edwards \& Bater 1992, Fredericksonet al 1997, Nogales et al 1995) estabilizándolos y transformándolos en un producto similar al humus denominado "vermicompost". En función a lo mencionado, el presente trabajo, evaluó el comportamiento de la lombriz roja (Eisenia spp.) bajo dos ambientes (Carpa Solar y Natural) con dos sustratos orgánicos (Estiércol de Vaca y Restos de Cocina) en predios de la Estación Experimental de la Carrera de Ingeniería Agronómica en la región de Nor Yungas, Departamento de La Paz-Bolivia. 
Materiales y métodos

La investigación se llevó a cabo en los predios de la Estación Experimental de la Carrera de Ingeniería Agronómica de la Unidad Académica Campesina de Carmen Pampa (UAC-CP), dependiente de la Universidad Católica Boliviana "San Pablo", situada en el Municipio de Coroico del Departamento de La Paz. La zona de vida corresponde a Bosque Húmedo Subtropical Premontano, altitud de 1815 m.s.n.m., con una precipitación media anual de $2185 \mathrm{~mm}$, humedad relativa de $79 \%$, temperatura media de $17.27^{\circ} \mathrm{C}$ y una velocidad media de viento $0.86 \mathrm{~m} \mathrm{~s}^{-1}$ (Estación Meteorológica UAC-CP, 1996-2004).

El experimento se condujo en dos ambientes: Carpa Solar (CS) y Ambiente Natural (AN). Los sustratos ensayados fueron: Estiércol de Vaca (EV) y Restos de Cocina (RC), materiales que fueron proporcionados por el Módulo Bovinos de la Carrera de Medicina Veterinaria-Zootecnia de la UAC-CP, se los dispusieron en camas o literas de $0.036 \mathrm{~m}^{3}$ de volumen. La cantidad de sustrato para cada unidad experimental fue de $13 \mathrm{~kg}$ de EV y $9 \mathrm{~kg}$ de RC, inoculándose $195 \mathrm{~g}$ de lombrices adultas (no cliteladas y cliteladas) del género Eisenia, que fueron proporcionadas por el Módulo de Lombricultura de la Carrera de Ingeniería Agronómica. La humedad de los sustratos se mantuvo alrededor del $80 \%$ HR mediante riegos periódicos. Las literas fueron cubiertas con hojas de cartón para evitar la desecación por los rayos solares.

Semanalmente y durante un periodo de vermicompostaje de 11 semanas, se obtuvieron muestras de $100 \mathrm{~g}$ de los sustratos ensayados (de cada tratamiento y repetición) para su evaluación, los parámetros biológicos de las lombrices, determinando el número, peso, grado de desarrollo y presencia de clitelo así como el número de cápsulas producidas. El sustrato de cada muestra pote fue volcado en una bandeja, las lombrices y cápsulas separadas manualmente. El procedimiento de pesado de lombrices fue similar al utilizado por otros autores (Serrano 2004, Sainz et al 2000, Benítez et al 2002) que consiste en separar manualmente las lombrices, lavarlas rápidamente en agua potable e introducirlas en un recipiente ligeramente húmedo. Antes de la pesada, cada lombriz es colocada unos segundos sobre papel de filtro que absorbe rápidamente el agua de lavado. Una vez pesadas, las lombrices y el sustrato fueron retornadas a sus literas. Diez cápsulas encontradas en cada muestra fueron colocadas en una placa petri entre 2 capas de papel de filtro humedecido e incubadas en oscuridad. En cada caja petri se colocó $5 \mathrm{~g}$ de sustrato para evitar la fuga de los recién nacidos una vez eclosionadas las cápsulas, posteriormente estos individuos fueron contados. Tras finalizar el periodo experimental, las lombrices, fueron retiradas de las literas y el producto final obtenido (vermicompost) fue homogeneizado, secado al aire, tamizándola en malla de $2 \mathrm{~mm}$. Las muestras fueron enviadas al laboratorio de suelos de South Dakota State University-USA donde se sometieron a análisis físico químico.

Finalmente, con el vermicompost obtenido se realizó una prueba o test de fitotoxicidad de acuerdo con la metodología descrita por Zucconi et al 1981b, Acosta et al 2006) se realiza un ensayo de germinación "in vitro" de semillas de puerro. Para éste fin se obtuvo un extracto acuoso 
de la muestra orgánica (relación 1:10) agitando la suspensión por 1 hora, luego se filtró el sobrenadante en papel filtro de $0.45 \mu \mathrm{m}$. En placa petri se dispuso un disco de papel de filtro sobre el cual se adicionó $1 \mathrm{~mL}$ del extracto embebiendo el papel y distribuyendo al azar 10 semillas de puerro, también se preparó un tratamiento control utilizando agua destilada. Las semillas fueron incubadas a $25^{\circ} \mathrm{C}$ en oscuridad durante 24 h luego de lo cual se detuvo la germinación añadiendo 1 $\mathrm{mL}$ de etanol al 50\%. Posteriormente, se contaron las semillas germinadas por placa, se determinó el porcentaje e índice de germinación (IG) de acuerdo a la siguiente expresión: \% germinación = (número de semillas germinadas en el extracto)/número de semillas germinadas en el control) x 100 .

Se adoptó un diseño bifactorial con tres repeticiones y los datos se sometieron al análisis de varianza (ANVA) con el nivel de significancia de $5 \%$ entre tratamientos.

\section{Resultados}

\section{Evolución del número de cápsulas}

Fig 1 Evolución del número de cocones en el sustrato Estiércol bovino en los dos ambientes ensayados

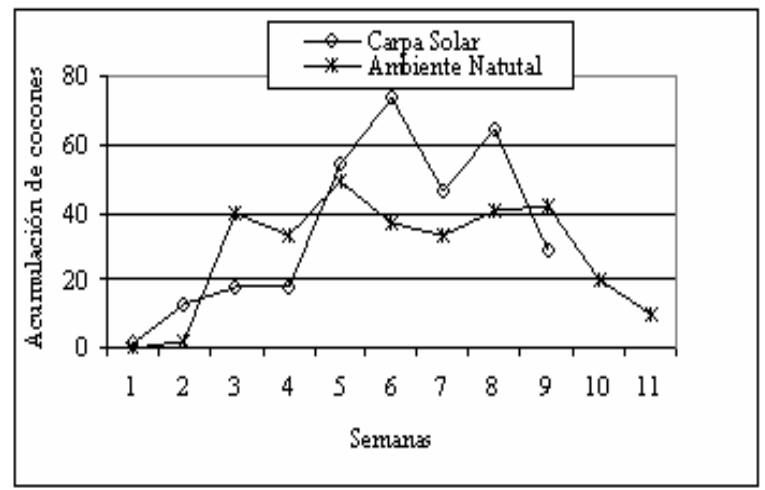

Fig 2 Evolución del número de cocones en el sustrato Restos de Cocina en los dos ambientes ensayados

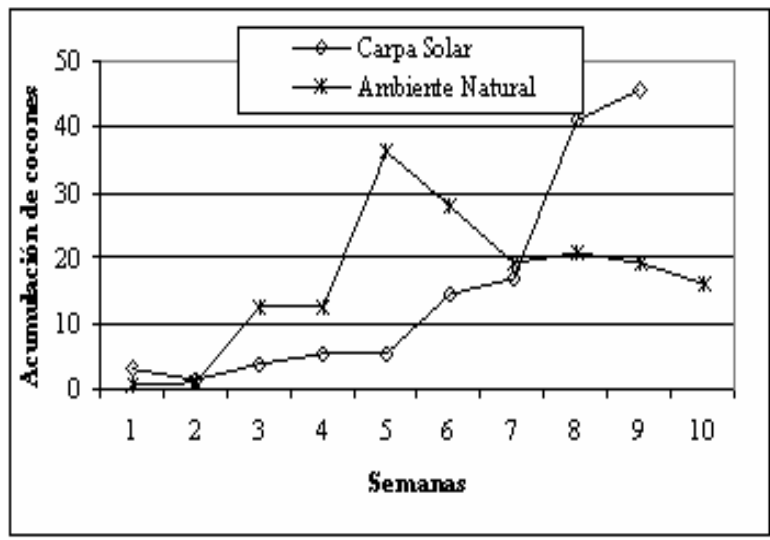

Tabla 1 Promedio del número de cápsulas en los dos ambientes y sustratos ensayados durante la octava semana de vermicompostaje

\begin{tabular}{ccccc}
\hline \multirow{2}{*}{ Parámetro } & \multicolumn{2}{c}{ Carpa Solar } & \multicolumn{2}{c}{ Ambiente Natural } \\
\cline { 2 - 5 } & EV & RC & EV & RC \\
\hline $\begin{array}{c}\text { Número de } \\
\text { cápsulas }\end{array}$ & 64 & 41 & 40 & 21 \\
\hline
\end{tabular}

CS. Carpa Solar, RC restos de Cocina.

Porcentaje de eclosión, número y peso de lombrices nacidas por cápsula

De las 10 cápsulas incubadas se obtuvieron los resultados mostrados en la tabla 2 , el análisis de varianza demuestra que no se encuentran diferencias para el factor ambiente y la interacción ambiente-sustrato, pero el factor sustrato tiene una diferencia $(p=0.001)$. Las diferencias entre sustratos se deben principalmente a la textura gruesa que presenta el sustrato restos de cocina el cual ocasiona mayor circulación del aire frio y caliente. 
Tabla 2 Promedio del porcentaje de eclosión de cápsulas y número de lombrices nacidas por cápsula en los dos ambientes y sustratos ensayados

\begin{tabular}{ccccc}
\hline \multirow{2}{*}{ Parámetro } & \multicolumn{2}{c}{ Carpa Solar } & \multicolumn{2}{c}{ Ambiente Natural } \\
\cline { 2 - 5 } & EV & RC & EV & RC \\
\hline $\begin{array}{c}\text { Porcentaje de eclosión } \\
\text { de cápsulas (\%) }\end{array}$ & 100 & 96 & 93 & 83 \\
\hline $\begin{array}{c}\text { Lombrices nacidas por } \\
\left.\text { cápsula ( } \mathrm{N}^{\circ}\right)\end{array}$ & 2.00 & 3.50 & 1.87 & 3.33 \\
\hline
\end{tabular}

CS. Carpa Solar, RC restos de Cocina.

\section{Número de lombrices no cliteladas}

Con respecto a la evolución del número de lombrices, en ambos sustratos (Figuras 3 y 4), se observa que la población de lombrices no cliteladas no sufre grandes variaciones hasta la tercera semana del periodo experimental. Esto se debe a que la población está compuesta por lombrices adultas en fase de postura; posteriormente, a partir de la cuarta semana hasta la culminación del experimento, se observa un aumento poblacional debido a la presencia de una nueva generación dominada por lombrices inmaduras (no cliteladas).

Fig 3 Evolución del número de lombrices no cliteladas en el sustrato Estiércol de Vaca en los dos ambientes ensayados

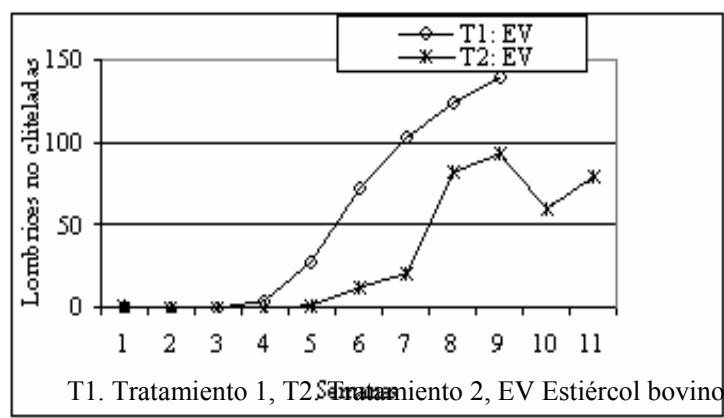

Fig 4 Evolución del número de lombrices no cliteladas en el sustrato Restos de Cocina en los dos ambientes ensayados

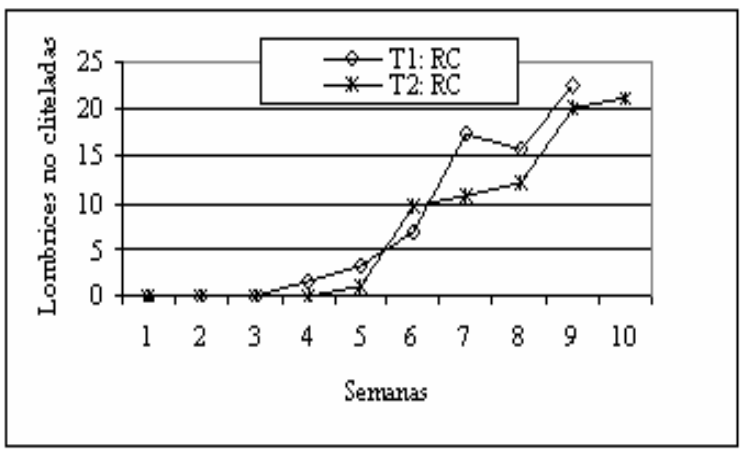

T1. Tratamiento 1, T2. Tratamiento 2, RC Restos de cocina.

Tabla 3 Promedio del número de lombrices no cliteladas en los dos ambientes y sustratos ensayados durante la octava semana de vermicompostaje

\begin{tabular}{ccccc}
\hline \multirow{2}{*}{ Parámetro } & \multicolumn{2}{c}{ Carpa Solar } & \multicolumn{2}{c}{ Ambiente Natural } \\
\cline { 2 - 5 } & EV & RC & EV & RC \\
$\begin{array}{c}\text { Número de } \\
\text { lombrices no } \\
\text { cliteladas }\end{array}$ & 123 & 16 & 82 & 12 \\
\hline
\end{tabular}

$\mathrm{RC}$ restos de Cocina. EV Estiércol bovino

En la octava semana se obtuvieron diferencias donde el ambiente CS y el sustrato EV resultaron los más adecuados para la población (Tabla 3).

El análisis de varianza determina que existe diferencias $(\mathrm{p}=0.001)$ para los factores ambiente $\mathrm{y}$ sustrato. 
Parámetros reproductivos y tasa de mortalidad de lombrices no cliteladas

Tabla 4 Resumen de los parámetros reproductivos y tasa de mortalidad de lombrices no cliteladas en los dos ambientes y sustratos ensayados

\begin{tabular}{|c|c|c|c|c|c|c|c|c|c|}
\hline \multirow{3}{*}{ Ambiente } & \multirow{3}{*}{ Sustrato } & \multicolumn{3}{|c|}{ Cápsulas } & \multirow{3}{*}{$\begin{array}{l}\text { Nacidos por } \\
\text { cápsula }\end{array}$} & \multirow{3}{*}{$\begin{array}{c}\text { Incremento } \\
\text { Actual }\end{array}$} & \multirow{2}{*}{\multicolumn{2}{|c|}{ Tasa de crecimiento }} & \multirow{3}{*}{$\begin{array}{c}\text { Mortalidad } \\
\%\end{array}$} \\
\hline & & \multirow{2}{*}{$\mathrm{Nr}$} & \multirow{2}{*}{$\begin{array}{c}\text { Eclosión } \\
\%\end{array}$} & \multirow{2}{*}{$\begin{array}{c}\text { Viables } \\
\mathrm{Nr}\end{array}$} & & & & & \\
\hline & & & & & & & $\begin{array}{c}\text { Incremento } \\
\text { Real }\end{array}$ & $\begin{array}{c}\text { Decremento } \\
\text { Actual }\end{array}$ & \\
\hline \multirow[t]{2}{*}{$\mathrm{CS}$} & $\mathrm{EV}$ & 64 & 100 & 64.00 & 2.00 & 123 & 128 & -5.00 & 3.90 \\
\hline & $\mathrm{RC}$ & 41 & 96 & 39.36 & 3.50 & 16 & 137 & -121.00 & 88.64 \\
\hline \multirow[t]{2}{*}{$\mathrm{AN}$} & $\mathrm{EV}$ & 40 & 93 & 34.20 & 1.87 & 82 & 69 & -13.00 & 18.68 \\
\hline & $\mathrm{RC}$ & 21 & 83 & 17.43 & 3.33 & 12 & 58 & -46.00 & 79.32 \\
\hline
\end{tabular}

Nr. Número, CS. Carpa Solar, AN. Ambiente Natural, EV. Estiércol Bovino, RC. Restos de Cocina

Tabla 4 se presenta un resumen de los parámetros reproductivos y la tasa de mortalidad de lombrices no cliteladas en ambos ambientes y sustratos ensayados.

\section{Número de lombrices cliteladas}

Tabla 5 Promedio del número de lombrices cliteladas en los ambientes y sustratos ensayados durante la octava semana de vermicompostaje

\begin{tabular}{ccccc}
\hline Parámetro & \multicolumn{2}{c}{ Carpa Solar } & \multicolumn{2}{c}{ Ambiente Natural } \\
\cline { 2 - 5 } & EV & RC & EV & RC \\
\cline { 2 - 5 } & 14 & 19 & 15 & 21 \\
$\begin{array}{c}\text { Número de } \\
\text { lombrices } \\
\text { cliteladas }\end{array}$ & & & & \\
\hline
\end{tabular}

EV Estiércol bovino, RC Restos de cocina

\section{Biomasa de lombrices}

La biomasa de lombrices durante la octava semana se presenta en la tabla 6. El análisis de varianza demuestra que los factores ambiente y sustrato tienen diferencias $(p=0.001)$, así como la interacción entre los dos factores ambientesustrato que presenta diferencias $(\mathrm{p}=0.001)$.
Tabla 6 Promedio de la biomasa de lombrices en los tratamientos $y$ sustratos ensayados durante la octava semana de vermicompostaje

\begin{tabular}{ccccc}
\hline \multirow{2}{*}{ Parámetro } & \multicolumn{2}{c}{ Carpa Solar } & \multicolumn{2}{c}{ Ambiente Natural } \\
\cline { 2 - 5 } & EV & RC & EV & RC \\
$\begin{array}{c}\text { Biomasa de } \\
\text { lombrices (g) }\end{array}$ & 1.410 & 0.185 & 0.705 & 0.173 \\
\hline
\end{tabular}

EV Estiércol bovino, RC Restos de cocina.

\section{Grado de fitotoxicidad}

Los resultados de la prueba fitoxicidad se muestran en la tabla 7 .

Tabla 7 Porcentaje de germinación de semillas de puerro con soluciones de vermicompost de los sustratos ensayados

\begin{tabular}{ccc}
\hline \multicolumn{1}{c}{ Parámetro } & \multicolumn{2}{c}{ Sustrato } \\
\cline { 2 - 3 } & EV & RC \\
\hline Porcentaje de germinación (\%) & 66 & 6.6 \\
\hline
\end{tabular}

EV Estiércol bovino, RC Restos de cocina. 
Análisis químico de los productos obtenidos

Tabla 8 Composición química del vermicompost obtenido de ambos sustratos ensayados

\begin{tabular}{lrcrc}
\hline \multicolumn{1}{c}{ Parámetro } & EV & Clasificación & RC & Clasificación \\
\hline Materia orgánica (\%) & 45.50 & Bajo & 29.70 & Bajo \\
Nitrógeno total (\%) & 2.31 & Medio & 2.45 & Medio \\
Fósforo P (ppm) & 220 & Bajo & 500 & Bajo \\
Potasio $\mathrm{K}_{\left(\mathrm{cmol}_{(+)} \mathrm{kg}^{-1}\right)}$ & 2.76 & Bajo & 27.43 & Alto \\
Calcio $\left.\mathrm{cmol}_{(+)} \mathrm{kg}^{-1}\right)$ & 4.14 & Bajo & 6.50 & Bajo \\
Magnesio $\left(\mathrm{cmol}_{(+)} \mathrm{kg}^{-1}\right)$ & 4.96 & Bajo & 5.00 & Bajo \\
Zinc $(\mathrm{ppm})$ & 32.00 & Bajo & 11.40 & Bajo \\
Cobre $(\mathrm{ppm})$ & 6.35 & Bajo & 1.30 & Bajo \\
pH & 7.10 & Neutro & 8.40 & Básico \\
\hline Conductividad eléctrica & 2.30 & Normal* & 8.00 & Alta \\
\hline
\end{tabular}

* No hay problemas de sales. EV Estiércol bovino, RC Restos de cocina.

La tabla 8 recoge los valores de los parámetros químicos determinados en los vermicompost obtenidos a partir de ambos sustratos.

\section{Discusión}

De acuerdo a los parámetros de Costa et al (1991), tanto el sustrato estiércol bovino (EV) como restos de cocina ( $\mathrm{RC}$ ) presentan valores bajos de materia orgánica. Elvira (1993) en un estudio similar obtuvo $56.4 \%$ para estiércol de vaca y $78.4 \%$ para residuos orgánicos domésticos. Por lo tanto, es claro que estos valores dependen de las características del material de partida.

Con respecto al nitrógeno, tanto $\mathrm{EV}$ como $\mathrm{RC}$ presentan valores medios. Sin embargo debe tomarse en cuenta que las lombrices asimilan la mayor parte de este elemento para realizar sus procesos filológicos, lo cual disminuye su concentración en el vermicompost obtenido (Hartenstein et al 1981).
El potasio se encuentra en altas concentraciones en RC debido a la abundancia de este elemento en los restos vegetales. Ya los valores de calcio y magnesio presentan bajas concentraciones al igual que el fósforo, lo cual puede deberse a la naturaleza de la materia prima y a las pérdidas por lixiviación o lavado por el riego periódico durante el proceso (Mamani et al 2008).

Los valores de microelementos como el cinc y cobre se presentan por debajo de los límites de tolerancia para uso agrícola de compost (Costa et al 1991, Nogales et al 1995).

Los valores de $\mathrm{pH}$ se encuentran en el rango neutro - alcalino. Estos pueden deberse a la presencia de elementos alcalinizantes que poseen los residuos domésticos, tales como sales $\mathrm{y}$ amonio liberado durante la fermentación de residuos ricos en nitrógeno. Tal comportamiento también fue observado por Serrano (2004) durante el vermicompostaje de residuos orgánicos urbanos. De acuerdo con Costa et al (1991) estos valores son compatibles con el crecimiento de la mayoría de los cultivos.

Con respecto a la Conductividad Eléctrica se determina que el sustrato EV no presenta problemas de sales, en cuanto que RC es considerado un sustrato fuertemente salino. Por tanto RC no es apto para utilizarse como enmienda orgánica en almácigos para semillas pequeñas, porque puede inhibir la germinación de las mismas y afectar el desarrollo inicial de las plantas. Además en grandes dosis, puede ocasionar un deterioro de la estructura el suelo. 
Se observa que en ambos sustratos el número de cápsulas no se incrementa significativamente hasta la segunda semana, pero de la tercera a la sexta se observa una fase de producción activa que después se torna decreciente tendencia similar fue observada por Benítez et al 2002, durante el vermicompostaje de estiércol mezclado con residuos agroindustriales. Se deduce que durante los primeros días, las lombrices sufrieron un estrés debido a su nuevo hábitat, lo cual se reflejó en una baja reproducción en ambos sustratos. Sin embargo, superada esta fase $y$ dada la disponibilidad de alimento, entraron en un periodo de producción óptima de cápsulas que decrece por agotamiento del sustrato alimenticio, en el caso de EV. Para RC se observa en el ambiente CS una elevada producción de cápsulas aún después de la séptima semana, probablemente porque aún había sustrato disponible y condiciones adecuadas para la reproducción. La diferencia más notable con respecto a la producción de cápsulas se observó en la octava semana, datos que se presentan en la tabla 1.

Las diferencias observadas en el anterior Cuadro se pueden atribuir a las condiciones ambientales altamente contrastantes que se tienen entre ambos ambientes donde para $\mathrm{CS}$ el promedio fue de $26.37^{\circ} \mathrm{C}$ en cuanto que en $\mathrm{AN} 17.81^{\circ} \mathrm{C}$. Por lo tanto en $\mathrm{AN}$, probablemente las bajas temperaturas afectaron el apareamiento de las lombrices y por lo tanto la probabilidad de fecundación y postura de huevos, conforme indican Reinecke \& Viljoen (1990).

Al igual que el número de cápsulas, las diferencias encontradas respecto a la eclosión pueden atribuirse a la diferencia de temperaturas entre 51 ambos ambientes. En este sentido en AN puede haber ocurrido un efecto negativo sobre las cápsulas, conforme indican Loehr et al (1985), Hernández et al (1997), quienes mencionan que a temperaturas bajas la albúmina de la cápsula tiende a coagularse dificultando de esta manera la alimentación de los embriones, llevando inclusive a su muerte. Con respecto al número de lombrices nacidas por cápsula, los mayores valores encontrados en RC muestran que puede haber influenciado el mayor contenido proteico de los residuos respecto al estiércol, conforme señala Bollo (2001). Por otro lado, los valores obtenidos se encuadran dentro de los rangos normales encontrados por Hernández et al (1997) que encontró entre 1 a 4 lombrices por cápsula, en cuanto que Elvira 1993, Serrano 2004, Palao 1999, encontraron un número promedio de 2 lombrices por cápsula.

Las diferencias encontradas respecto a la mayor población en CS y EV respecto a $\mathrm{AN}$ y $\mathrm{RC}$ muestran que la mayor temperatura ambiente promedio de CS influyó considerablemente en el aumento de la población de lombrices no cliteladas; resultado corroborado por Hernández et al (1997), quién encontró que a una temperatura promedio de $31^{\circ} \mathrm{C}$, un adecuado ritmo de crecimiento y reproducción de estos organismos.

Por otro lado la granulometría del sustrato RC puede haber sido decisiva para las grandes diferencias entre EV y RC en ambos ambientes. Es decir en RC las lombrices inmaduras pueden no haber encontrado un tamaño ideal de las partículas para alimentarse como en EV, lo cual afectó considerablemente su desarrollo. Este aspecto ya fue observado por Serrano (2004). Por su parte 
Palao (1999) quien durante el vermicompostaje de residuos sólidos y lodos encontró elevados índices de mortalidad dada la tendencia del sustrato a desarrollar condiciones anaeróbicas.

Como se observa en la tabla 4, el ambiente CS presenta una tasa de mortalidad menor para el sustrato EV, conforme ya se mencionó, probablemente debido a las mejores condiciones ambientales. Analizando el efecto del sustrato RC en ambos ambientes resaltan las elevadas tasas de mortalidad que provocó este sustrato en ambos ambientes (superior al 70\%). Entre los varios factores analizados, se puede atribuir este efecto a la elevada Conductividad Eléctrica encontrada en RC que alcanzó los $8.0 \mathrm{dS} \mathrm{m}^{-1}$ (Fuertemente salino), lo cual puede haber sido determinante en la mortalidad de las lombrices. La sensibilidad de las lombrices a la salinidad también es resaltada por Lee (1985), quien describe que la cutícula corporal de estos organismos es permeable al agua y a los iones en disolución; por los que altas concentraciones de sales ocasionan un desequilibrio osmótico mortal. Los resultados obtenidos están de acuerdo con los de Nogales et al 1995, 1999, Hernández et al 1997, quienes encontraron mayor mortalidad en rangos de 3.0 a $5.37 \mathrm{dS} \mathrm{m}^{-1}$. Por otro lado Elvira (1993) recomienda que es importante que la conductividad eléctrica se mantenga en valores inferiores a $2.0 \mathrm{dS} \mathrm{m}^{-1}$, sobre todo para garantizar la supervivencia de las lombrices juveniles.

Conforme se observa en la tabla 5 anterior, existen diferencias mínimas con respecto al número de lombrices cliteladas en ambos sustratos. De manera similar Serrano (2004) determinó que en sustrato Estiércol Vacuno, el número total de lombrices cliteladas se mantuvo uniforme a lo largo el periodo experimental. Esto se explica porque las lombrices desarrollaron su cóitelo probablemente entre la semana 3 y 6 , que es cuando se obtiene la máxima producción de cápsulas. Una vez alcanzado el desarrollo máximo, las lombrices tendieron a perder el cóitelo; conforme mencionan Sainz et al (2000), lo cual puede suceder cuando se inicia la fase de agotamiento de nutrientes del sustrato.

Comparativamente los datos del tabla 6 muestran un mayor peso promedio de las lombrices inoculadas en EV en ambos ambientes. Los valores obtenidos fueron similares o incluso superiores a los observados en otros estudios, utilizando diferentes tipos de substratos orgánicos (Edwards \& Bater 1992, Elvira 1993, Frederickson et al 1997, Haimi 1990). Las diferencias observadas entre los dos sustratos ensayados pondrían de manifiesto que $\mathrm{EV}$ es el sustrato con los recursos nutritivos $\mathrm{y}$ granulometría adecuada para el desarrollo de las lombrices. No obstante las limitaciones químicas y físicas del sustrato RC pueden mantener una población viable de estos organismos.

Los resultados de la tabla 7 muestran diferencias la fitotoxicidad del vermicompost obtenido de RC en ambos ambientes (Carpa solar y ambiente natural). El estiércol de vaca resulta ser el menos toxico y el sustrato RC se constituye como el más toxico. El motivo fundamental radicaría en la presencia de compuestos de naturaleza orgánica (ácidos orgánicos de bajo peso molecular, sales y otros), los cuales tienen un marcado carácter inhibidor de la germinación de semillas (Saviozzi et al 1993, Moreno et al 2000). El proceso de 
vermicompostaje desarrollado, no fue eficaz para reducir este efecto.

El proceso de vermicompostaje con el uso de la lombriz roja californiana (Eisenia spp.) es posible de ser optimizado en condiciones de Carpa Solar. Los residuos orgánicos domésticos (restos de cocina) presentan una agresividad para las lombrices del género Eisenia spp., por lo que afectaron su crecimiento y reproducción adecuada. El desarrollo de las mismas fue mayor cuando se utilizó estiércol vacuno, el cual constituye el medio natural de crecimiento de estos organismos.

Los residuos de cocina son materiales orgánicos que por su naturaleza química (salinidad elevada) y tamaño de partícula presentaron dificultad para ser degradados por las lombrices. Sin embargo, permitieron el mantenimiento de una población viable que pudo reproducirse.

Los productos obtenidos al final del proceso de vermicompostaje no cumplieron con la mayoría de las especificaciones sobre contenidos mínimos en principios activos. Además, el vermicompost de RC mostró una elevada fitotoxicidad.

La tecnología de vermicompostaje de residuos orgánicos domésticos debe ser desarrollada y optimizada para mitigar sus características negativas como sustrato.

\section{Agradecimientos}

Los autores agradecen a la Unidad Académica Campesina Carmen Pampa (UAC-CP), Ingeniería Agronómica.

\section{Literatura citada}

Acosta Y, Cayama J, Gómez E, Reyes N, Rojas D, García H.Respiración microbiana y prueba de fitotoxicidad en el proceso de compostaje de una mezcla de residuos orgánicos. Multiciencias. 2006;6:220-227.

Benítez E, Sainz H, Melgar R, Nogales R. Vermicomposting of a lignocellulosic waste from olive oil industry: a pilot scale study. Waste Management \& Research. 2002;20:134142.

Bollo E. Lombricultura: una alternativa de reciclaje. SOBOC. Quito. 2001:179pp.

Costa F, García C, Hernández T, Polo A. Residuos orgánicos urbanos: manejo y utilización. CSICCEBAS. Murcia, España. 1991;181p.

Edwards CA, Bater JE. The use of earthworms in environmental management. Soil Biol. Biochem. 1992;24(812);1683-1689.

Elvira C. Vermicompostaje de residuos orgánicos. $\mathrm{Su}$ aplicación a lodos generados por la industria papelera. Tesis der doctorado. Universidad de Santiago de Compostela. España. 1993. 289pp.

Frederickson J, Butt KR, Morris RM, Daniel C. Combining vermiculture with traditional green waste composting systems. Soil Biology \& Biochemistry. 1997;19:725-730.

Haimi J. Growth and reproduction of the compostliving earthworm Eisenia andrei and E.fetida.Rev Ecol Biol Sol. 1990;27:415-421.

Hartenstein R, Hartenstein F. Physicochemical changes effected in activated sludge by the earthworm Eisenia foetida. Ed. Herat worm ecology from Darwin to vermiculture, Londres. 1981;10(3);377-392. 
Hernández J, Rincón $\mathrm{M}$, Jiménez R. Comportamiento de la lombriz roja (Eisenia sp.) bajo condiciones de clima cálido. Maracaibo, Venezuela. 1997;14:387-392.

Lee KE. Earthworms. Their ecology and relationships with soils and land use. Academic Press. Sydney. 1985: 411pp.

Loehr RC, Neuhauser EF, Malecki R. Factors affecting the vermistabilization process.Temperature, moisture content and polyculture. Water Res Tech. 1985;19(10):1311-1317.

Mamani-Mamani GE, Mamani-Pati F. Red Worm Behavior (Eisenia spp) With Different Types of Organic Residues in Carmen Pampa, La Paz - Bolivia. In 2008 International Annual Meeting Abstracts. ASA-CSSA- SSSA, Houston, TX. USA. 2008.

Moreno R, Benítez E, Melgar R, Polo A, Gomez $\mathrm{M}$, Nogales R. Vermicomposting as an alternative for reusing by-products from the olive oil industry. Fresenius Environ Bull. 2000;9:1-8.

Nogales R, Elvira C, Benítez E, Gallardo-Lara F. Uso agrícola de compost y vermicompost de basuras urbanas (I): Procesos, madurez y calidad de los productos. Residuos 1995;26:5357.

Nogales R, Elvira C, Benítez E, Thompson R, Gomez M. Feasibility of vermicomposting dairy biosolids using a modified system to avoid earthworm mortality. J Environ Sci \& Health. Part B. 1999;34(1);151-169.
Palao IL. Biotransformación de residuos sólidos urbanos (basura y lodo), con lombrices de tierra Eisenia foetida. UMSA. Facultad de Ciencias Puras y Naturales. Tesis de Maestría en Ecología y Conservación. La Paz, Bolivia. 1999. 120pp.

Reinecke AJ \& Viljoen SA. The influence of worm density on growth and cocoon production of the compost worm Eisenia foetida (Ohgochaeta). Rev Ecol Biol Sol. 1990;27(2):221-230.

Sainz H, Benítez E, Melgar R, Álvarez R, Gómez $\mathrm{M}$, Nogales R. Biotransformación $\mathrm{y}$ valorización agrícola de subproductos del olivar, orujos secos y extractados mediante vermicompostaje. Edafología. 2000;7(2):103111.

Saviozzi A, Riffaldi R, Levi-Minzi R, Scagnozzi A, Vanni G. Decomposition of vegetationwater sludge in soil. Biores Technol.1993;44:223-228.

Serrano CT. Evaluación de procesos de vermicompostaje para el tratamiento de residuos sólidos urbanos de la localidad de Tihuanacu. Tesis de Licenciatura. Universidad Catolica Boliviana. La Paz, Bolivia. 2004. 53p.

Sistema Nacional de Capacitación en Saneamiento Básico. S.N.C.S.B. Operación y Mantenimiento de Rellenos Sanitarios. Santa Cruz. 14 al 16 de Septiembre de 2000. 200pp.

Zucconi F, Forte M, Monaco A, De Bertoldi M. Biologicalevaluation of compost maturity. BioCycle. 1981b;22(4):27-29. 